Jesuis entré ensuite, près d'Hermonville, dans unecarrière depierres, encore en exploitation, dont les galeries profondes pénètrent de plainpied dans le flane d'un coteau. A la différence de la carrière précédente, elle abritait peu de $\boldsymbol{S}$. libatrix, mais les $\boldsymbol{A}$. granitella y pullulaient. Elles se tenaient de toute part posées sur les parois, très loin de l'entrée, et jusqu'a une distance d'environ quatre-vingts mètres; elles s'arrêtaient d'ailleurs, avant d'atteindre l'extrémité des galeries. Nous avons capturé de plus une Triphosa dubitata L., Phalène que j'ai vue aussi associée aux Acrolepia dans la grotte du Loup. Enfin les Insectes étaient en outre représentés en cet endroit par de nombreux Diptères, et par quelques Coléoptères, parmi lesqucls je citerai le Catops depressus Murr.

Les A. granitella cherchent dans ces souterrains un abri pour passer l'hiver. On les y retrouve dans les premiers jours du printemps, mais elles fréquentent aussi ces refuges en d'autres saisons : c'est en íté, dans la seconde quinzaine du mois d'aoủt 1888, que je les ai observées à Lourdes. Il est curieux que l'on rencontre toujours exclusivement cette espèce, et pas d'autres du même genre ou de la même famille.

Les $\boldsymbol{S}$ c. libatrix et $\boldsymbol{T r}$. dubitata, qui habitent avec elle, sont un peu partout les hôtes les plus ordinaires des caves et des sonterrains. Les mœurs de ces deux Lépidoptères sont bien connues; mais, quant i l'A. granitella, ses habitudes cavernicoles n’ont été, à ma connaissance, signalées nulle part, en dehors de la note très brève que j’ai publiée en 1890.

\title{
Deux espèces nouvelles de Nepticula [LÉP.]
}

par J. DE JoAnNis.

La faune lépidoptérique française est encore bien peu étudiée, notamment en ce qui concerne les plus petites espèces; un grand nombre se trouvent en France que les catalogues ne signalent point, bien d'autres également s'y rencontrent qui sont encore inédites. Les belles recherches de M. P. Chrétien ont concouru, plus que toutes autres, depuis plusieurs années, à mettre ce fait en évidence. Comme nouvelle preuve, voici deux espèces inédites de Nepticula, vivant sur des plantes absolument vulgaires, l'une sur la Ronce, l'autre sur le Prunellier, découvertes à Vannes par mon frère, L. de Joannis, et sur les- 
quelles nous possédons actuellement tous les documents nécessaires pour les faire connaitre.

Nepticula erythrogenella, nov. sp. - Exp. alarum : 4,ö-

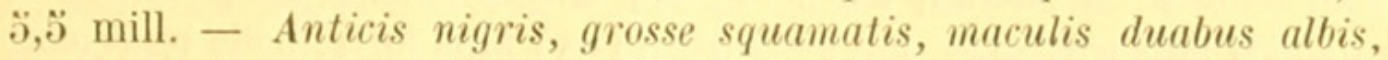
nitidis, triangularibus, altera ad costam ante medium, altera ad marginem internum post medium, utraque versus marginem externum incurvata, earumque apicibus quandoque squamis albis religatis. Ciliis divisis, medietate externa alba. Posticis griseis. Capillis rufo-brunneis, antennis griseis, brevibus, conchula albida subflavescenti. Pedibus griseis. Abdomine, thorace, collari griseo-fuscescentibus.

Supérieures noires, a écailles grossières, avec deux taches blanches, luisantes, mais sans reflet métallique; l'une à la còte, immédiatement avant le milieu, l'autre au bord interne, immédiatement après le milieu ; toutes deux sont infléchies vers l'extérieur et, parfois, leurs pointes sont reliées par une petite trainée courbe d'écailles blanches. Frange nettement divisée en deux parties, la moitié extérieure blanche, la partie basale noire, terminée par une ligne de grosses écailles noires. Inférieures grises. Tète à poils d'un brun roussâtre, antennes grises, courtes, avec les œillères d'un blanc un peu jaunitre. Pattes grises. Abdomen, thorax, collier d'un gris noiritre.

62 exemplaires.

Chenille gris légèrement verdâtre, translucide, avec les trois avantderniers anneaux teintés de jaune, mais paraissant parfois noiràtres par suite de la coloration des organes internes; canal brun roussâtre, parfois très visible. Tète brun noiràtre, bord de l'écusson thoracique noir, une série de taches ventrales, longitudinales, noires. Chemine dans sa galerie le dos contre l'épiderme inférieur.

Cocon elliptique, un peu irrégulier, blanc dans les premières heures, puis tourne au rose vineux et devient a la longue brun violacé. Chrysalide facilement, contre les feuilles ou les parois de la boite.

La chenille paraît de la fin de septembre a la fin de novembre, minant les feuilles de Ronce. Les éclosions, en captivité, ont eu lieu du 8 juin au 22 juillet; elle n'a qu'une génération. Se trouve à millions aux environs de Vannes, dans les Ronces de toutes les haies.

Espèce absolument distincte de toutes celles qui vivent sur la Ronce par le dessin de l'aile supérieure, composé de deux taches séparées, non métalliques, et non d'une bande continue métallique ; ce qui ne per. met pas de la confondre notamment avec $\boldsymbol{N}$. rubivora W $\mathbf{k}$., la seule des espèces de la Ronce qui ait la frange divisée. - Appartient', comme 
aspect général, au groupe d'agrimoniæ Frey, angulifasciella Frey, atricollis Stt.

La mine n'est pas moins remarquable et tout à fait caractéristique. Elle débute en suivant les nervures, faisant parfois un petit coude quand il faut traverser une nervure un peu plus forte, mais reprenant vite sa direction, puis se replie sur elle-mème, longeant son premier parcours assez longtemps, deux ou plusieurs fois de suite, complètement remplie par les excréments; puis, dans une seconde période, s’élargit en plaque irrégulière oì les excréments sont disposés sans ordre, accumulés ici, dispersés ailleurs. Mais, ce qui la rend tout à fait remarquable, et d'où j’ai tiré son nom, c'est la coloration d'un rouge rose violacé, parfois fort vif, qu'elle détermine dans la région qu'elle occupe, et qui la dissimule merveilleusement, à cause de l'analogie que présente cette tache rouge avec les taches naturelles de vieillissement autumnal des feuilles de Ronce $\left(^{1}\right)$.

N. spinosella, nov. sp. - Exp. alarum : 4 mill. - Anticis nigris, grosse squamatis, fascia metallescente pallide aurata immediate ante medium, ad costam leviter versus basim incurvata. Ciliis divisis, medietate postrema albida. Posticis griseis, capillis rufescentibus, antennis griseis, brevibus, conchula alba. Pedibus griseis. Abdomine, thorace, collari griseo-fuscescentibus.

Supérieures noires, à écailles assez grossières, avec une bande d'or pâle (parfois argentée) partant du bord interne immédiatement avant le milieu et très légèrement incurvée vers la base. Frange divisée, la portion extérieure blanchâtre, assez courte. Inférieures grises. Tète i poils roussâtres, antennes grises, courtes, avec les æillères blanches. Pattes grises; abdomen, thorax, collier gris noiràtre.

26 exemplaires.

Chenille vert d'eau pâle, tant qu'elle n'est pas adulte, jaune clair quand elle est adulte et qu'elle sort de la feuille; les deux ou trois derniers segments teintés d'orangé; la tète rougeâtre; l'écusson notablement plus rouge que chez N. plagicolella, ce qui la fait paraître au premier aspect verdìtre très pâle avec les deux extrémités rougeâtres. Les anneaux marqués en dessous de taches noires allongées. Chemine dans sa galerie le dos contre l'épiderme inférieur.

Le cocon est gris jaunâtre argileux, souvent un peu plus large à une

(1) M. Mirande, maitre de conférences à la Faculté des Sciences de Montpellier, a bien voulu examiner le mécanisme de ce rougissement et compte présenter une note à ce sujet à l'Académie des Sciences. 
extrémité qu'à l'autre, un peu irrégulier. Chrysalide facilement en captivité, comme la précédente.

La chenille parait du commencement de septembre a la fin d'octobre, minant les feuilles de Prunellier. Les éclosions ont eu lieu, en captivité, du 21 juin au 10 juillet. Elle semble avoir deux éclosions; des mines vides et déjà très vieilles ont en effet été observées en juillet, elles ne pouvaient provenir de l'apparition d'automne, il devait donc y avoir eu une éclosion de chenilles quelque temps auparavant. Elle est répandue dans toute la région autour de Vannes, cependant elle semble avoir des prélérences pour les lieux un peu ombragés, pour le coté nord des haies.

Sa mine, au début, est en ligne contournée, irrégulière, tantòt plus étroite, tantòt plus large, aux deux tiers remplie par les excréments; puis cette galerie, toujours irrégulière et sinueuse, parfois se déroule dans la feuille, d'autres fois et le plus souvent se replie sur elle-mème, mais finit toujours par une plage irrégulière un peu élargie, n'ayant aucunement la forme arrondie de la plaque blanche de plagicolella. Elle se distingue d'ailleurs de celle-ci par beaucoup d'autres détails. Notamment la galerie initiale de plagicolella est toujours d'un noir vif, celle de spinosella, lorsqu'elle se dessèche, parait d'un violacé pâle, et, dans la petite plage terminale, les excréments sout disposés en ligne irrégulière, se recoupant ou s'éparpillant.

Complètement différente des deux espèces du Prunellier : d'abord par la position de la bande avant le milieu et par la frange divisée en deux parties; ensuite elle se distingue immédiatement de $\boldsymbol{N}$. prunetorum par la couleur de la tète, roussàtre au lieu d'ètre noire, et l'absence de toute teinte dorée au bord interne près de la base, et de plagicolella par l'absence de teinte pourpre sur l'aile. Appartient au groupe de rubivora $\mathrm{Wk}$., arcuatella $\mathrm{H} . \mathrm{S}$., etc.

La mine et la chenille sont d'ailleurs absolument différentes de celles des deux autres espèces du Prunellier, qui sont assez connues pour qu'il ne soit pas nécessaire de noter ici les différences.

\title{
Description de deux espèces nouveiles de Lepidoptères algeriens
}

\author{
par Ch. Oberthür.
}

C'est en dressant l'inventaire des Lépidoptères algériens connus vers 1875 que nous commençâmes la publication de nos Études d'En- 


\section{$2 \mathrm{BHL}$ Biodiversity Heritage Library}

Joannis, Joseph de. 1908. "Deux espèces nouvelles de Nepticula (Lep )." Bulletin de la Société entomologique de France 1907, 326-329.

View This Item Online: https://www.biodiversitylibrary.org/item/38671

Permalink: https://www.biodiversitylibrary.org/partpdf/4582

\section{Holding Institution}

Smithsonian Libraries

\section{Sponsored by}

Smithsonian

\section{Copyright \& Reuse}

Copyright Status: NOT_IN_COPYRIGHT

This document was created from content at the Biodiversity Heritage Library, the world's largest open access digital library for biodiversity literature and archives. Visit BHL at https://www.biodiversitylibrary.org. 